\title{
Article \\ Fluid Supply and Feeding Practices in Cooled Asphyxiated Newborns
}

\author{
Mona Markus ${ }^{1}$, Stamatios Giannakis ${ }^{1}$, Maria Ruhfus ${ }^{2}$, Anja Stein ${ }^{2} \mathbb{D}$, Axel Heep ${ }^{3}$, Thorsten Plagemann ${ }^{3}$, \\ Peter Jahn ${ }^{4}$, Thomas Hoehn ${ }^{1}$, Ursula Felderhoff-Mueser ${ }^{2}$ and Hemmen Sabir ${ }^{5,6, *}$
}

1 Department of General Pediatrics, Neonatology and Pediatric Cardiology, Medical Faculty and University Hospital Duesseldorf, Heinrich-Heine-University Duesseldorf, 40225 Duesseldorf, Germany; mona.markus@hotmail.de (M.M.); stamatios.giannakis@gmail.com (S.G.); Thomas.Hoehn@med.uni-duesseldorf.de (T.H.)

2 Department of Pediatrics I/Neonatology, University Hospital Essen, University Duisburg-Essen, 45147 Essen, Germany; maria.ruhfus@gmail.com (M.R.); Anja.Stein@uk-essen.de (A.S.); Ursula.Felderhoff@uk-essen.de (U.F.-M.)

3 Department of Paediatrics, Elisabeth Children's Hospital, University of Oldenburg, 26133 Oldenburg, Germany; Heep.Axel@klinikum-oldenburg.de (A.H.); Plagemann.Thorsten@klinikum-oldenburg.de (T.P.)

4 Department of Neonatology, Children's Hospital Leverkusen, 51375 Leverkusen, Germany; peter.jahn@klinikum-lev.de

5 Department of Neonatology and Pediatric Intensive Care, Children's Hospital University of Bonn, 53127 Bonn, Germany

6 German Centre for Neurodegenerative Diseases (DZNE), 53175 Bonn, Germany

* Correspondence: hemmen.sabir@ukbonn.de

Citation: Markus, M.; Giannakis, S.; Ruhfus, M.; Stein, A.; Heep, A.; Plagemann, T.; Jahn, P.; Hoehn, T.; Felderhoff-Mueser, U.; Sabir, H. Fluid Supply and Feeding Practices in Cooled Asphyxiated Newborns. Children 2021, 8, 899. https:// doi.org/10.3390/children8100899

Academic Editor: Sari A. Acra

Received: 13 September 2021

Accepted: 6 October 2021

Published: 9 October 2021

Publisher's Note: MDPI stays neutral with regard to jurisdictional claims in published maps and institutional affiliations.

Copyright: (c) 2021 by the authors. Licensee MDPI, Basel, Switzerland. This article is an open access article distributed under the terms and conditions of the Creative Commons Attribution (CC BY) license (https:/ / creativecommons.org/licenses/by/ $4.0 /)$.

\begin{abstract}
Therapeutic hypothermia (TH) for $72 \mathrm{~h}$ is the standard treatment to reduce neurological deficits in term newborns with hypoxic-ischemic encephalopathy. There is a large variability regarding nutritional supply during TH treatment in asphyxiated newborns. We performed a retrospective multicentre study in four level I (highest level of care in Germany) NICUs, including 135 asphyxiated term newborns undergoing TH. We analyzed enteral and parenteral nutritional supply during and after TH. We correlated nutritional supply with risk factors for encephalopathy, $\mathrm{pH}$, Sarnat score, mechanical ventilation, seizures, and sedation. A total of 120 of 135 neonates received enteral nutritional supply within the first $24 \mathrm{~h}$, and the majority of children were fully enterally fed within the first 10 days. The grade of encephalopathy and mechanical ventilation had a significant influence on the amount of enteral fluids $(p=0.01)$, whereas the $\mathrm{pH}$ and appearance of seizures did not affect the amount of nutritional supply significantly. Furthermore, we did not observe any correlation between enteral intake and abdominal complications such as necrotizing enterocolitis. We observed a large variability of feeding regimes in the four participating NICUs. Early enteral feeding among newborns undergoing TH was performed in each NICU and was well tolerated without increased rates of complications.
\end{abstract}

Keywords: perinatal asphyxia; hypoxic-ischemic encephalopathy; therapeutic hypothermia; enteral nutrition; parenteral nutrition

\section{Introduction}

Hypoxia-ischemia (HI) following perinatal asphyxia may cause neonatal encephalopathy (NE) in term infants. Clinically NE is associated with the onset of seizures and may lead to short and long-term problems such as multi-organ failure, mental retardation, abnormal feeding, or respiratory distress [1]. Therapeutic hypothermia (TH) for $72 \mathrm{~h}$ started within the first $6 \mathrm{~h}$ after birth is the treatment of choice reducing death or neurological disabilities in newborns suffering hypoxic-ischemic encephalopathy (HIE) [2].

Due to a lack of evidence, there are no clear guidelines regarding nutritional supply during TH in newborns with HIE following perinatal asphyxia. Enteral and also parenteral 
nutrition is an important topic in asphyxiated children undergoing $\mathrm{TH}$ for $72 \mathrm{~h}$. Enteral nutrition is physiological, and parenteral nutrition increases the risk for infection [3]. A U.K.-wide e-mail survey reports that only 31 percent of neonatal intensive care units (NICUs) follow guidelines concerning nutritional supply during $\mathrm{TH}$ [4]. Only 59 percent of the participating hospitals started enteral feeding during cooling [4]. Therefore, there is great variability in terms of feeding regimes. It has been shown that minimal enteral nutrition in cooled asphyxiated newborns is feasible [5]. Furthermore, it was found that minimal enteral nutrition under TH causes a shortened length of hospital stay and time until full oral feeds are achieved [6]. One of the main reasons to withhold enteral feedings during $\mathrm{TH}$ is the anxiety for the development of side effects, for example, necrotizing enterocolitis (NEC). In contrast to the proposed understanding that a delay of enteral feeding might prevent NEC, a study from the U.K. reported that a delayed onset of enteral feeding did not reduce the risk of developing NEC in preterm infants [7]. In contrast, it resulted in a delay of time to full enteral feeds [7].

Furthermore, the early introduction of enteral nutrition also bears several advantages. It has a positive effect on gut-barrier function, immune function and reduces bacterial translocation [8]. A delayed start of enteral nutrition results in an increasing rate of chronic lung disease, intestinal inflammation, and an increased risk of morbidity [9]. In addition, both hyperglycemia and hypoglycemia can lead to poor neurological outcomes in cooled asphyxiated newborns [10]. Therefore, it is important to establish an optimal feeding regime for cooled asphyxiated newborns.

In the present study, we collected data from four level I (highest level of neonatal care) NICUs in Germany with regard to enteral and parenteral nutrition in asphyxiated newborns with HIE undergoing TH. The aim was to identify similarities and differences in the nutritional fluid regime in order to recognize possible pros and cons of early initiation of enteral feeding.

\section{Materials and Methods}

\subsection{Data Collection}

Data from 135 term ( $\geq 37+0$ weeks of gestation) neonates born between 2008 and 2019 from 4 level 1 NICUs in Germany were retrospectively collected and analyzed. The study was approved by the local ethical committees. Thirty-eight newborns were born at $(n=6)$ or transferred to $(n=32)$ the NICU of the University Hospital Oldenburg; thirtynine newborns were born at $(n=24)$ or transferred to $(n=15)$ the NICU of the University Hospital Essen; 38 were born at $(n=24)$ or transferred to $(n=14)$ the NICU of the Children's Hospital Leverkusen, and 20 were born at $(n=11)$ or transferred to $(n=9)$ the NICU of the University Hospital Duesseldorf. Parts of data from this cohort have been previously published [11,12].

All newborns were treated with whole-body hypothermia for $72 \mathrm{~h}$, started within the first $6 \mathrm{~h}$ after birth, maintaining a rectal temperature of $33-34{ }^{\circ} \mathrm{C}$ due to NE of presumed HI. Newborns fulfilled the entry criteria for hypothermia therapy as proposed by the large randomized-controlled cooling trials (Apgar score $\leq 5$ and/or ongoing resuscitation at $10 \mathrm{~min}$, abnormal blood gases with a $\mathrm{pH}<7.0$ or base deficit $\geq 16 \mathrm{mmol} / \mathrm{L}$ as the immediate criteria for perinatal asphyxia, abnormal neurologic examination as the second criterion, and moderate or severe abnormalities on amplitude-integrated encephalography (aEEG) or seizures as the third). The presence of seizures was defined clinically and/or by aEEG. We used the aEEG pattern classification as in previous studies to define abnormal EEG and detection of seizures [13].

Data for gestational age, sex, birthplace (inborn/outborn), birth weight, survival, Apgar scores at 5 and $10 \mathrm{~min}$, need for mechanical ventilation, lowest $\mathrm{pH}-$, base excessand lactate values before initiation of cooling, degree of encephalopathy before initiation of cooling (Sarnat score), onset and treatment of subclinical or clinical seizures before and during the cooling period were collected. The lowest $\mathrm{pH}$ and base excess are defined as the 
first gas obtained within the first hour after birth. In 90\% of cases, it was obtained from cord gas.

Data of enteral and parenteral nutritional supply was collected until newborns received full enteral feeds, and total nutritional supply was collected over a period of 7 days. Enteral nutrition included human breast milk or replacement formula, and parenteral nutrition included all i.v. carbohydrates, amino acids, and fats. The total nutritional intake consisted of all parenteral and enteral nutritional supplies. NICU1 aimed an initial enteral fluid intake of $7 \mathrm{~mL} / \mathrm{kg} / \mathrm{d}$ and raised the fluids by $41 \%$ per day during TH. Children in NICU2 started with an enteral fluid intake of $3 \mathrm{~mL} / \mathrm{kg} / \mathrm{d}$ and increased supply daily by $35 \%$ during TH. NICU3 started with $10 \mathrm{~mL} / \mathrm{kg} / \mathrm{d}$ and raised enteral fluids by $44 \%$ per day, and NICU4 started with an initial enteral fluid intake of $7 \mathrm{~mL} / \mathrm{kg} / \mathrm{d}$ and increased fluids by $34 \%$ daily under $\mathrm{TH}$.

The analysis of enteral nutritional supply included time of initiation of enteral feeding in hours, feeding situation at discharge, days of central and peripheral venous access, and weight gain until discharge. Weight gain was defined as a gain of weight per individual day. Data on cumulative morphine dosage and duration of morphine administration was also collected.

Two NICUs with the greatest difference in terms of the amount of nutritional supply were compared. We focused on the comparison between the amount of total, enteral and parenteral nutrition.

Data for total, enteral and parenteral fluid intake were available for all children except those who died within the first $24 \mathrm{~h}(n=6)$. No infant was excluded from the study.

\subsection{Data Analysis}

We correlated nutritional fluid intake with the following risk factors: lowest $\mathrm{pH}$ within the first hour after birth $(<6.8 ; 6.8-7.0 ;>7.0)$, Sarnat scores (Sarnat grade $1,2,3)$, seizures (seizures vs. no seizures) and ventilatory status (mechanical ventilation vs. no mechanical ventilation) within the first week of life. Pearson's correlation was used to compare each individual parameter against enteral fluid intake.

Data of cumulative morphine doses were collected in $\mathrm{mg} / \mathrm{kg} / \mathrm{d}$. In two centers, the average cumulative morphine dose for the whole period was documented; in the other two centers, the average for each day was individually calculated. One center used fentanyl instead of morphine.

Total, enteral, and parenteral fluid supply and change in weight were additionally compared between the two centers with the most divergent regime.

SPSS 26 (SPSS, Chicago, IL, USA) was used for statistical analysis. The statistical significance level was 5\%. Mann-Whitney $\mathrm{U}$ test was used to analyse nonparametric data between the different centers included within the study. Individual repeated measures were analyzed for nutritional intake over time. Descriptive statistics were used for all variables to identify the median and interquartile range as also the mean and standard deviation for continuous variables and the frequency distributions for categorical variables.

\section{Results}

\subsection{General}

We included 135 newborns in our study ( $n=66$ males, $n=69$ females). Mean gestational age was $39 \pm 2$ weeks. A total of 65 children were inborn, and 70 were transferred to the respective NICU within the first six hours of life. Median (range) APGAR scores after 1, 5 , and 10 min were $1(0-10), 4(0-10)$, and $6(0-10)$. A total of 16 of 135 children died. A total of six children died within the first $24 \mathrm{~h}$ and eight children within the first week. All descriptive patient data are presented in Table 1. 
Table 1. Demographic data and perinatal risk factors of the total study cohort $(n=135)$.

\begin{tabular}{cc}
\hline & Total $(\boldsymbol{n = 1 3 5 )}$ \\
Birth weigth (mean \pm SD g) & $3284 \pm 817$ \\
Gender (n, \% male) & $66(49)$ \\
Gestational Age ( mean \pm SD days) & $269 \pm 5$ \\
APGAR Score 5 min median (range) & $4(0-10)$ \\
APGAR Score 10 min median (range) & $6(0-10)$ \\
worst pH (mean \pm SD) & $6.735 \pm 1.2$ \\
worst Base Excess (mean \pm SD) & $17.6 \pm 14.6$ \\
worst Lactate Level (mean \pm SD) & $11.6 \pm 7.2$ \\
HIE Grade before HT ( $n=$ mild, $n=$ moderate, $n=$ severe) & $65(48)$ \\
Inborn (n, \%) & $16(12)$ \\
Death (n, $\%)$ & mild, $75=$ moderate, 29= severe
\end{tabular}

\subsection{Total, Enteral, and Parenteral Fluid Supply}

All NICUs started with a nutritional supply within the first $24 \mathrm{~h}$ of life at a mean $( \pm \mathrm{SD})$ total volume of $55.6 \mathrm{~mL} / \mathrm{kg} / \mathrm{d}( \pm 14)$ and increased the supply daily during cooling by a mean $( \pm \mathrm{SD})$ of $11 \mathrm{~mL} / \mathrm{kg} / \mathrm{d}( \pm 0.5)$ (Table 2$)$. The largest rise of total nutritional supply was observed after the rewarming phase (day 4 to 5$)$ (mean \pm SD: $16 \pm 0.75 \mathrm{~mL} / \mathrm{kg} / \mathrm{d}$ ). After the fifth day of life, the total nutritional fluid increase was reduced to a mean $( \pm \mathrm{SD})$ of $6 \mathrm{~mL} / \mathrm{kg} / \mathrm{d}( \pm 0.46)$. After the first week of life, the newborns received twice as much total fluid intake compared to the first day of life (day 7, mean (SD) $113 \mathrm{~mL} / \mathrm{kg} / \mathrm{d}( \pm 21.25)$ (Figure 1A)).

Table 2. Nutritional supply data of the total study cohort $(n=135)$.

\begin{tabular}{cc}
\hline & Total $(\boldsymbol{n = 1 3 5 )}$ \\
\hline Start oral feeding (mean \pm SD h) & $11 \pm 12.1$ \\
Days until full enteral feeds (mean \pm SD days) & $9.2 \pm 4.4$ \\
Cummulative Morphin dosage (mean \pm SD mg/kg $/ \mathrm{h})$ & $0.06 \pm 0.05$ \\
Duration Morphin (mean \pm SD h) & $84 \pm 38$ \\
Days central venous access (mean \pm SD) & $7 \pm 4.8$ \\
Days peripheral venous access (mean \pm SD) & $7 \pm 4$ \\
Weight gain until discharge (mean \pm SD g) & $232 \pm 273$ \\
Feeding situation at dischrge & $100=$ fully breast fed, $17=$ gastric tube, $17=$ death, \\
& $1=$ not assignable
\end{tabular}

Enteral feeding with human breast milk was started in 120 neonates within the first $24 \mathrm{~h}$ (mean (SD) $11 \mathrm{~h}(6 \mathrm{~h})$ ). All centers started enteral feeding within the first $72 \mathrm{~h}$, and the majority of newborns were fully enterally fed within the first 10 days (mean $( \pm \mathrm{SD})$ : 9.2 ( \pm 4.4 days $)$ ).

Enteral feeding was started with a mean $( \pm S D)$ enteral volume $(E N)$ of $7 \mathrm{~mL} / \mathrm{kg} / \mathrm{d}$ $( \pm 2.5)$ and increased until full enteral feeds were achieved around day 12 with a mean $( \pm \mathrm{SD})$ volume of $86 \mathrm{~mL} / \mathrm{kg} / \mathrm{d}( \pm 24)$. The highest amount of enteral nutritional fluid intake was observed after 2 weeks (day 14, mean (SD) $93.42 \mathrm{~mL} / \mathrm{kg} / \mathrm{d}( \pm 28)$ ). The amount of enteral feeding exceeded the amount of parenteral feeding (PN) after 8 days (EN: $63.76 \mathrm{~mL} / \mathrm{kg} / \mathrm{d} \pm 21, \mathrm{PN}: 59.96 \mathrm{~mL} / \mathrm{kg} / \mathrm{d} \pm 17$ ) (Figure 1B).

Newborns received a higher amount of parenteral nutritional supply than enteral nutritional supply within the first week of life. The amount of enteral feeding exceeded the amount of parenteral feeding (PN) after 8 days. 


\section{Comparison between total, enteral and parenteral food supply}
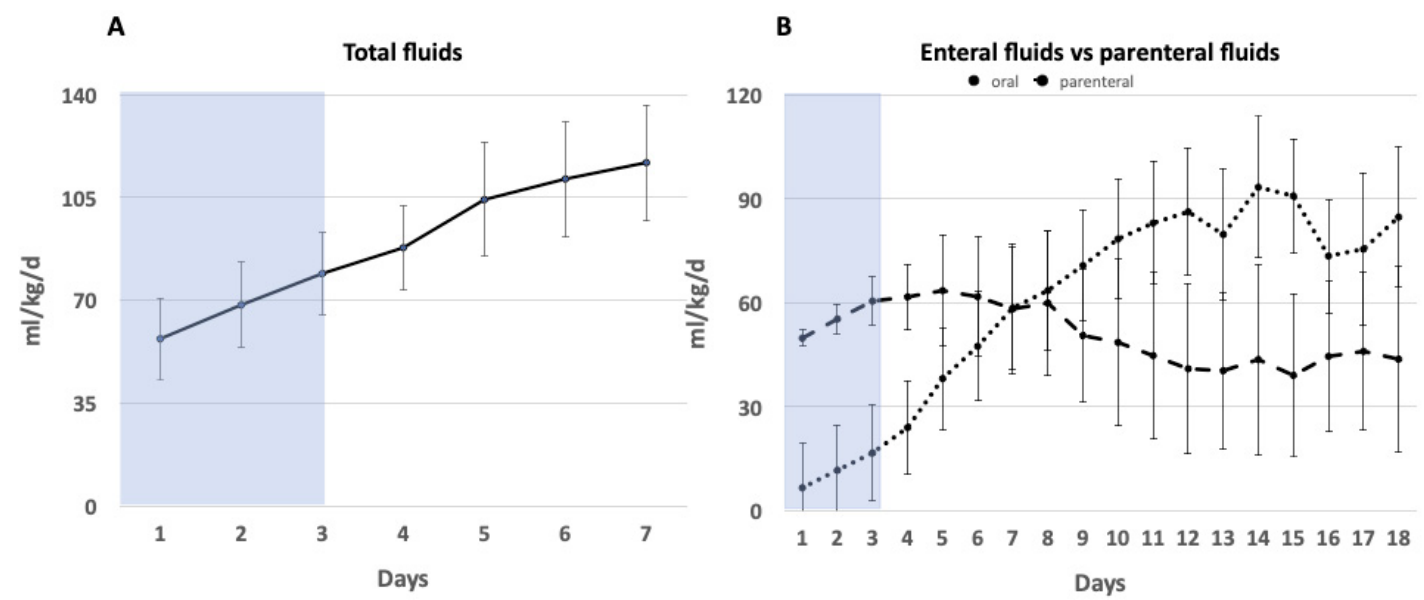

Figure 1. Comparison between total, enteral, and parenteral nutritional supply. Data presented in mean $\mathrm{mL} / \mathrm{kg} / \mathrm{d}$ $(+/-\mathrm{SD})$. Blue shaded box represents time of TH. (72 h). (A) Total nutritional fluid intake of the first week of life for children undergoing therapeutic hypothermia given in $\mathrm{mL} / \mathrm{kg} / \mathrm{d}$. All children received total nutritional supply within the first 7 days and increased supply also during cooling. (B) Comparison between enteral and parenteral nutritional supply in children undergoing $\mathrm{TH}$ over a period of 18 days given in $\mathrm{mL} / \mathrm{kg} / \mathrm{d}$.

\subsection{Weight Gain}

Daily weight gain was analyzed within the first 20 days (Figure 2). A daily weight gain was observed within the analyzed period (mean (SD) weight day 1 vs. day 20 (day 1 $3275 \mathrm{~g}( \pm 420)$ vs. day $203664 \mathrm{~g}( \pm 627))$.

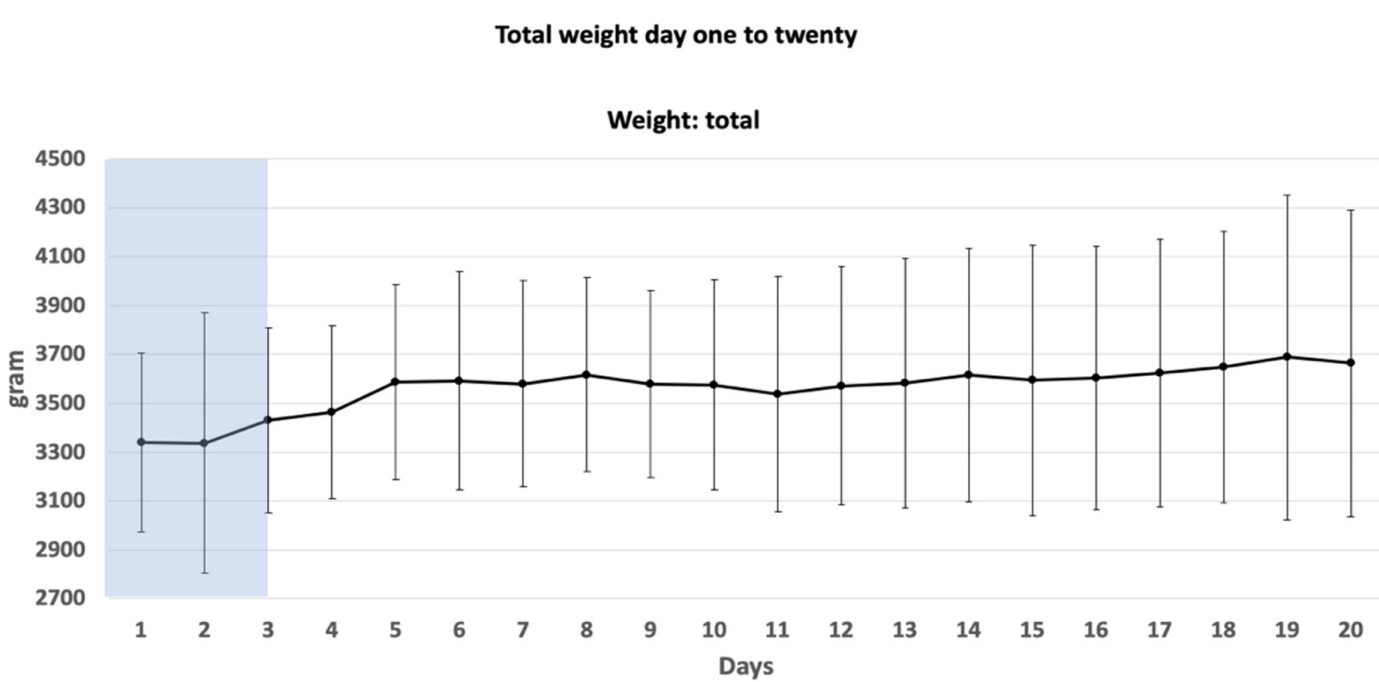

Figure 2. Change in weight of all newborns within the first 20 days after birth. Data presented in mean gram ( \pm SD). Blue shaded box represents time of $\mathrm{TH}(72 \mathrm{~h})$.

\subsection{Risk Factors}

Newborns with a lower grade of encephalopathy (Sarnat grade 1) received a significantly higher amount of enteral nutrition within the first 6 days compared to children with a higher grade of encephalopathy (Sarnat grade 3) $(p=0.01)$. 
Comparing children with Sarnat score grade 2 and 3, children with Sarnat score grade 3 received a significantly lower amount of enteral fluid supply within the first 5 days $(p=0.02)$ (Figure 3C).

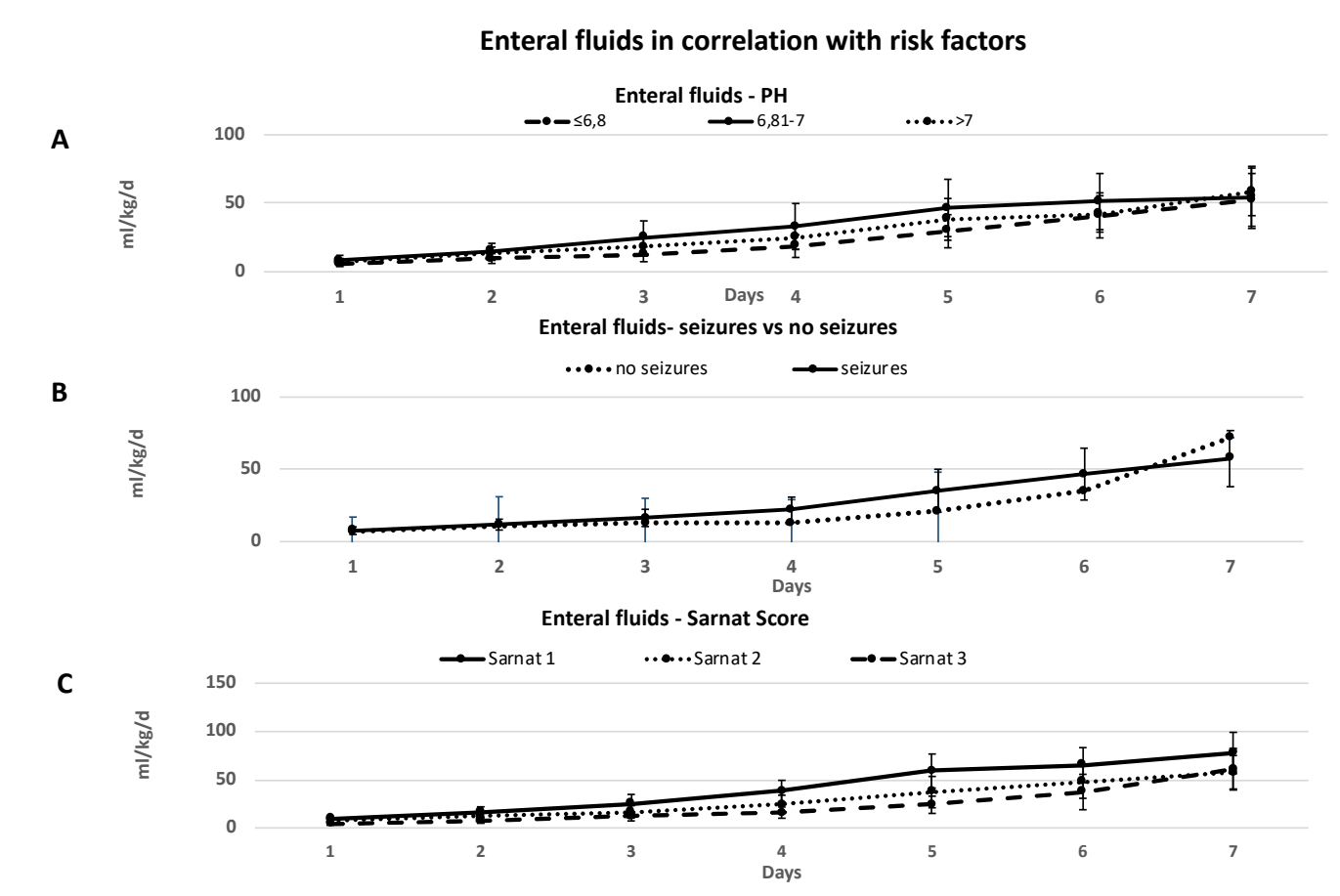

Enteral fluids: mechanical ventilation vs no mechanical ventilation

D

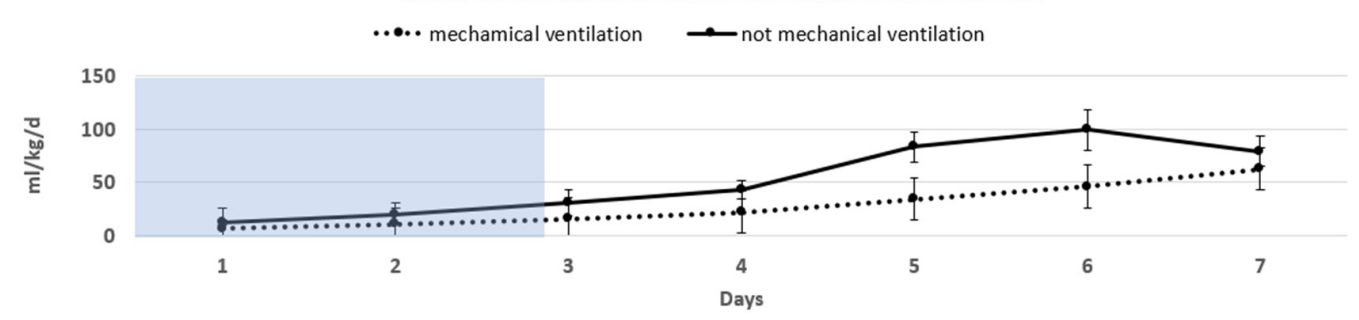

$\mathbf{E}$

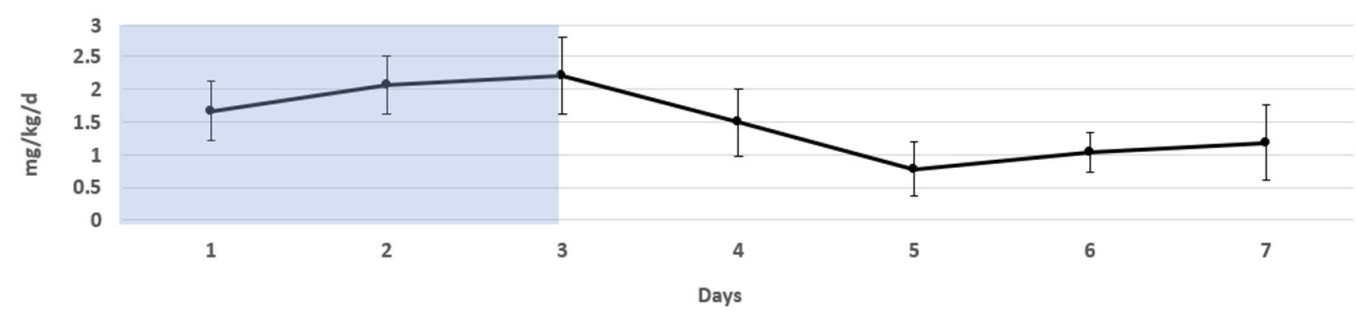

Figure 3. Amount of enteral nutritional fluid supply in the first week of life in correlation with risk factors. Blue shaded box represents time of $\mathrm{TH}(72 \mathrm{~h})$. (A,B) Lowest $\mathrm{pH}$ after birth $(\mathbf{A})$ and appearance of seizures (B) did not influence the amount of enteral fluids significantly. (C) Grade of encephalopathy (Sarnat score) had a significant influence on the amount of enteral nutritional fluid supply. Neonates with a higher grade of encephalopathy received a significantly lower amount of enteral nutritional fluids. (Sarnat score grade 1 vs. $3 p=0.01$, Sarnat grade 2 vs. $3 p=0.02$ ). (D) Mechanical ventilation was associated with a significantly lower amount of nutritional intake during the first 6 days $(p=0.01)$ (D). (E) Cumulative morphine dose over a period of 7 days given in $\mathrm{mg} / \mathrm{kg} / \mathrm{d}$. After $\mathrm{TH}$ was finished $(72 \mathrm{~h})$ cumulative morphine dose was strongly decreased. Data presented in mean $\mathrm{mg} / \mathrm{kg} / \mathrm{d}( \pm \mathrm{SD})$. 
Mechanical ventilation was associated with a significantly lower amount of enteral nutritional intake during the first 6 days $(p=0.01)$. With the exception of day 2 , newborns without mechanical ventilation received twice as much enteral nutrition in comparison to children with mechanical ventilation in the first 6 days (mean (SD) day 1 and 6 (day 1: $12.2 \mathrm{~mL} / \mathrm{kg} / \mathrm{d}( \pm 2.8)$ and day $6: 99.4 \mathrm{~mL} / \mathrm{kg} / \mathrm{d}( \pm 18.7)$ vs. day $1: 6.1 \mathrm{~mL} / \mathrm{kg} / \mathrm{d}( \pm 2.4)$ and day 6: $45.9 \mathrm{~mL} / \mathrm{kg} / \mathrm{d}( \pm 16.1)$ ) (Figure 3D).

First $\mathrm{pH}$ after birth and the onset of seizures did not influence nutritional intake significantly (Figure 3A,C).

Comparing the cumulative morphine doses, we observed that the median (range) duration of morphine exposure was 84 (11-288) h (Table 2). Most children were intubated during morphine administration. We did not observe any abdominal complications in children receiving morphine. Morphine was started continuously i.v. at a mean (SD) dose of $0.07 \mathrm{mg} / \mathrm{kg} / \mathrm{h}( \pm 0.0048)$. During cooling, the mean (SD) morphine dose was increased to $0.09 \mathrm{mg} / \mathrm{kg} / \mathrm{h}( \pm 0.008)$ and decreased after the end of cooling by a mean (SD) dosage of $0.03 \mathrm{mg} / \mathrm{kg} / \mathrm{h}( \pm 0.008)$ (day 5) (Figure 3E).

\subsection{Comparison of the Two NICUs with the Largest Differences}

Comparing the two NICUs with the largest differences in amount of total, enteral and parenteral nutritional supply, we observed the following differences: NICU 1 started with a significantly higher amount of total nutritional supply in contrast to NICU 2 (NICU 1 mean (SD) $70 \mathrm{~mL} / \mathrm{kg} / \mathrm{d}( \pm 17)$ ) vs. NICU 2 mean (SD) $39.7 \mathrm{~mL} / \mathrm{kg} / \mathrm{d}( \pm 7), p<0.005)$ (Table 3). Furthermore, newborns in NICU 1 received a significantly higher amount of total nutritional supply within the first 4 days (mean NICU1 $86.03 \mathrm{~mL} / \mathrm{kg} / \mathrm{d}( \pm 14) \mathrm{vs}$. NICU2 $51.53 \mathrm{~mL} / \mathrm{kg} / \mathrm{d}( \pm 10), p<0.05)$.

Table 3. Descriptive and nutritional supply data from newborns of two different NICUs.

\begin{tabular}{|c|c|c|}
\hline & NICU $1(n=38)$ & NICU $2(n=38)$ \\
\hline Birth weight (mean \pm SD g) & $3615 \pm 915$ & $3256 \pm 636$ \\
\hline Gender (n, \% male) & $20(54)$ & $21(55)$ \\
\hline Gestational Age (mean \pm SD days) & $279 \pm 46$ & $272 \pm 22$ \\
\hline APGAR Score 5 min median (range) & $4(0-8)$ & $4(0-10)$ \\
\hline APGAR Score 10 min median (range) & $6(0-10)$ & $6(0-10)$ \\
\hline lowest $\mathrm{pH}($ mean $\pm \mathrm{SD})$ & $6.9 \pm 0.23$ & $6.9 \pm 0.18$ \\
\hline worst Base Excess (mean \pm SD) & $14.8 \pm 10.4$ & $19.7 \pm 19$ \\
\hline worst Lactate Level (mean \pm SD) & $9 \pm 7$ & $12.1 \pm 5.7$ \\
\hline $\begin{array}{l}\text { HIE Grade before HT ( } n=\text { mild, } n=\text { moderate, } \\
n=\text { severe) }\end{array}$ & $10=$ mild, $26=$ moderate, $2=$ severe & $\begin{array}{c}6=\text { mild }, 23=\text { moderate } \\
\quad 9=\text { severe }\end{array}$ \\
\hline Inborn $(\mathrm{n}, \%)$ & $6(16)$ & $24(63)$ \\
\hline Death $(n, \%)$ & $5(13)$ & $3(8)$ \\
\hline Start oral feeding (mean \pm SD h) & $11.5 \pm 5.6$ & $16.4 \pm 22$ \\
\hline Days until full enteral feeds (mean \pm SD days) & $8 \pm 2.5$ & $12.8 \pm 4.4$ \\
\hline Cumulative Morphin dosage (mean $\pm \mathrm{SD} \mathrm{mg} / \mathrm{kg} / \mathrm{h}$ ) & $0.04 \pm 0.02$ & $0.12 \pm 0.05$ \\
\hline Duration Morphin (mean $\pm \mathrm{SD} h)$ & $94 \pm 37$ & $92 \pm 27$ \\
\hline Days central venous access (mean $\pm \mathrm{SD}$ ) & $6.5 \pm 3.1$ & $11.14 \pm 5.13$ \\
\hline Days peripheral venous access (mean \pm SD) & $8 \pm 5$ & $9 \pm 5$ \\
\hline Weight gain until discharge(mean \pm SD g) & $251 \pm 360$ & $306 \pm 242$ \\
\hline Feeding situation at discharge & $\begin{array}{c}27=\text { fully breast fed, } 6=\text { gastric tube, } \\
5=\text { death, }\end{array}$ & $\begin{array}{c}33=\text { fully breast fed, } \\
1=\text { gastric tube, } 3=\text { dead, } 1=\text { not } \\
\text { assignable }\end{array}$ \\
\hline
\end{tabular}

While the nutritional intake in children in NICU 1 only increased until the second day of life and then continuously given between $90 \mathrm{~mL} / \mathrm{kg} / \mathrm{d} \pm 15$ and $95 \pm 16 \mathrm{~mL} / \mathrm{kg} / \mathrm{d}$ (minimum, maximum), the nutritional fluid intake for children in NICU 2 increased daily. The increase in newborns in NICU 2 was higher when $\mathrm{TH}$ was finished $(+8 \mathrm{~mL} / \mathrm{kg} / \mathrm{d}$ vs. +17.7 mL/kg/d) (Figure 4A). 
A

Total, enteral and parenteral food supply: Center 1 vs Center 2
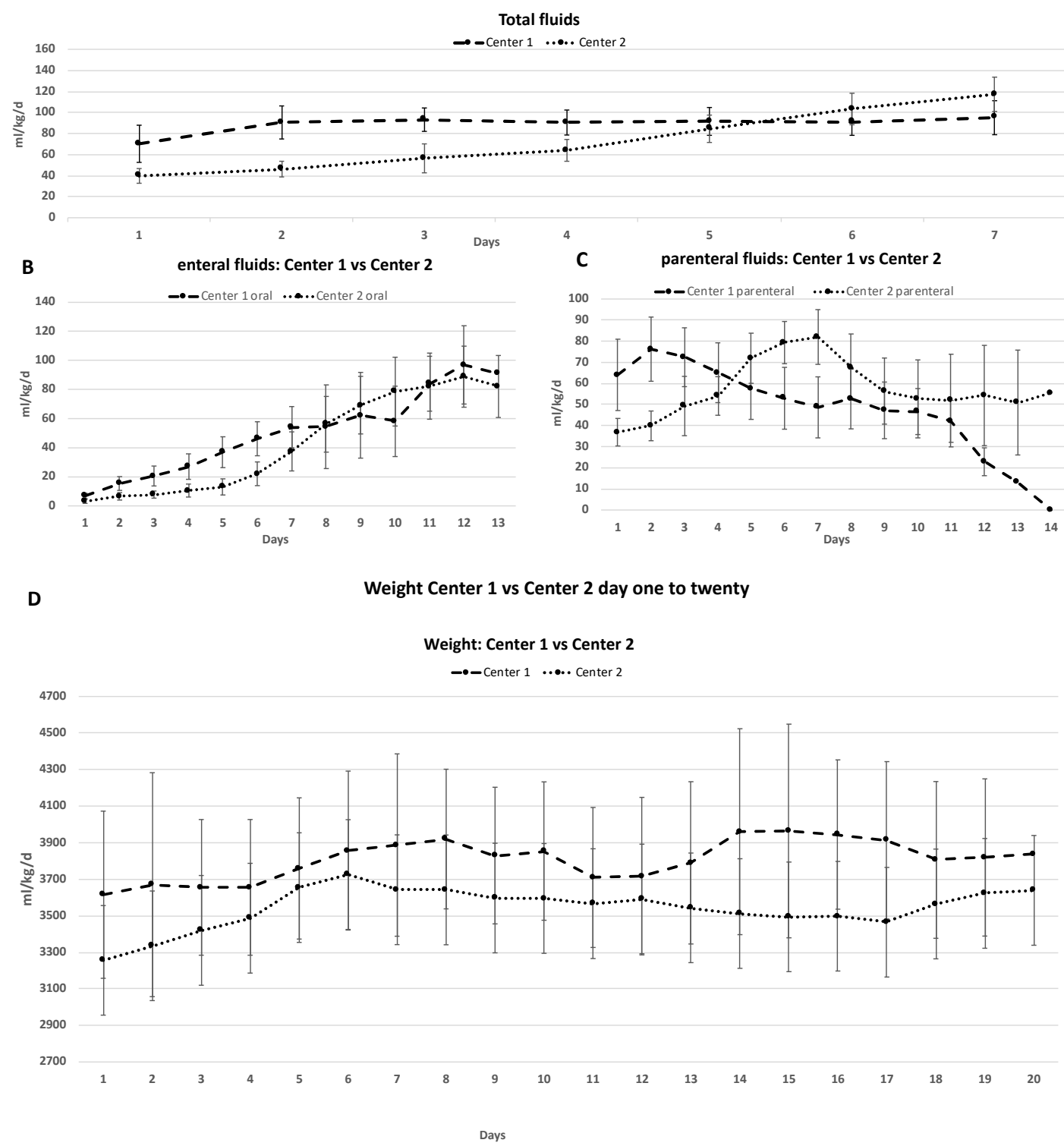

Figure 4. Comparison of total, enteral, and parenteral nutritional supply in two different NICUs. Data presented in mean $\mathrm{mL} / \mathrm{kg} / \mathrm{d}( \pm \mathrm{SD})$. Blue shaded box represents time of TH $(72 \mathrm{~h})$. (A-C) NICU 1 received a significantly higher amount of total (A), enteral (B), and parenteral (C) nutrition. (D) Comparison of change in weight over a period of 20 days. Data presented in mean gram $( \pm \mathrm{SD})$.

We also observed differences in enteral nutrition. NICU 1 started with a significantly higher amount of enteral nutrition $(6.85 \mathrm{~mL} / \mathrm{kg} / \mathrm{d}( \pm 1.8) \mathrm{vs} .3 \mathrm{~mL} / \mathrm{kg} / \mathrm{d}( \pm 1.3))$, and children received a significantly higher amount of enteral nutrition within the first week $(p<0.05)$. During the cooling phase, children in NICU 1 received more than twice the amount of enteral nutrition compared to NICU 2. (NICU 1: $14.23 \mathrm{~mL} / \mathrm{kg} / \mathrm{d}( \pm 4.4)$, NICU 2: $5.76 \mathrm{~mL} / \mathrm{kg} / \mathrm{d}( \pm 2.1)$. NICU 1 showed a lower cumulative morphine dose $(0.04 \pm 0.02 \mathrm{mg} / \mathrm{kg} / \mathrm{h})$, an earlier start of enteral nutrition $(11.5 \mathrm{~h} \pm 5.6 \mathrm{vs} .16 .4 \mathrm{~h} \pm 22)$, an earlier timepoint when newborns were fully enterally fed (8 days \pm 2.5 vs. 12.8 days \pm 4.4 ) and a shorter period of central venous access ( 6 days \pm 3 vs. 11 days \pm 5 ). Both NICUs showed no increase in the rate of complications such as sepsis or necrotizing enterocolitis (Figure 4B).

Focusing on parenteral nutrition (PN) between the two NICUs, NICU 1 started PN with a significantly higher amount than NICU 2 (NICU1 64.1, $\pm 16.9 \mathrm{~mL} / \mathrm{kg} / \mathrm{d}$ vs. NICU2 
$36.86, \pm 6.5 \mathrm{~mL} / \mathrm{kg} / \mathrm{d}, p=0.001)$. Newborns in NICU 1 received a significantly higher amount of parenteral nutrition during the cooling phase and from day five to seven. NICU 1 increased parenteral fluid supply until day $2(76.22 \mathrm{~mL} / \mathrm{kg} / \mathrm{d}, \pm 15.2)$ and then decreased parenteral fluid intake until day 14 . NICU 2 increased parenteral fluid supply until day 7 $(81.97 \mathrm{~mL} / \mathrm{kg} / \mathrm{d}, \pm 12.97)$ and decreased parenteral fluid supply afterward. (Figure $4 \mathrm{C}$ ).

\subsection{Complications during Enteral Feeding}

We observed no abdominal complications such as necrotizing enterocolitis in children receiving enteral feeds during and after cooling. Sepsis (defined as positive blood culture) was found in 25 of 135 children; however, we did not observe any correlation to enteral feeding supplies.

\subsection{Situation at Discharge}

A total of 100 children were discharged fully breastfed and 17 (13\%) children with a gastric tube. A total of $16(12 \%)$ patients died, and for one patient, the discharge situation was not reported. Breastfeeding was started on day 7 (mean $( \pm 2.5)$ and mean time to full breastfeeding was achieved after 13 days $( \pm 5)$.

\section{Discussion}

This observational multicentre study examined the enteral and parenteral nutritional fluid supply of asphyxiated neonates undergoing TH in four major NICUs in Germany. We found that all neonates started total nutritional supply, and the majority started enteral nutrition within the first $24 \mathrm{~h}$ after birth. All NICUs started enteral feeding during the cooling period, within the first $72 \mathrm{~h}$ after birth. Different factors such as mechanical ventilation, morphine dose, and grade of encephalopathy had a significant influence on the amount of enteral fluid supply. We did not find any association between enteral feeding and abdominal complications during the cooling period or after rewarming. Comparing the two NICUs with the largest differences in feeding and fluid practices, we observed meaningful differences in amount of total, enteral, parenteral nutritional supply, and morphine dose. This was independent of the level of encephalopathy.

Therapeutic hypothermia (TH) for $72 \mathrm{~h}$ is the standard therapy in newborns $\geq 36+0$ weeks of gestation suffering from moderate or severe hypoxic-ischemic encephalopathy (HIE) following perinatal asphyxia [14]. TH improves neurologic outcomes at two years, reduces mortality, and does not increase major disabilities [2,14,15].

Enteral and parenteral nutrition is an important aspect during TH, as metabolism may be reduced during $\mathrm{TH}$. Therefore, it is surprising that there are no clear guidelines regarding nutritional supply in cooled asphyxiated newborns. A U.K.-wide e-mail survey reported that only $31 \%$ of participating NICUs follow clear guidelines concerning nutritional supply during TH [4]. Our own national guideline in Germany has no recommendation regarding the nutritional supply during cooling [16], and also, guidelines from other countries do not provide clear advice regarding enteral nutrition and recommend a more individual decision for each child [17].

In our study, we found that the nutritional regime in asphyxiated cooled newborns was not standardized in general but that neonates were managed very individually with large differences between different NICUs. The decision process was not always concise, and we were unable to comprehend some of the decisions.

The key reason to withhold enteral nutrition in newborns during TH remains the potential risk of developing NEC. The pathogenesis of NEC is not completely understood. NEC is assumed to arise from multifactorial reasons such as genetics, abnormal microbial colonization, intestinal immaturity, and immunoreactive intestinal mucosa [18]. Based on the belief that enteral feeding is thought to cause NEC, it is often started rather restrictively during TH. On the other hand, enteral nutrition in newborns with breast milk is physiological and has positive effects such as stimulation of the intestinal motility, support of the 
intestinal immune system, protection of the intestinal mucosa, and stimulation of intestinal hormones.

Despite the risk of developing NEC in our study, enteral feeding was started in 120 of 135 neonates within the first $24 \mathrm{~h}$ of life, and all children started enteral feeding during $\mathrm{TH}$ (within the first $72 \mathrm{~h}$ of life). We did not observe any case of NEC in our cohort. Furthermore, the amount of enteral and also parenteral nutrition was increased in all investigated NICUs during TH. Other NICUs in Europe showed different management in commencing enteral feeding. An e-mail survey from the U.K. reported that almost $60 \%$ of participating NICUs started enteral feeding during TH and rewarming [4]. Moreover, a retrospective cohort study from U.K. and Sweden showed that in the Swedish cohort, 91\% of newborns were enterally fed during cooling, and in the U.K. cohort, just 31\% [5]. Although the beginning of enteral nutrition was managed differently, none of the infants in our study, as well as in the U.K. and Swedish cohort, developed abdominal complications such as NEC [5].

Comparing the two NICUs with the greatest difference in nutritional fluid intake during TH in our study, we found that NICU 1 started with almost twice the amount of total nutritional fluid intake compared to NICU 2. Children in NICU 2 received a significantly higher morphine dose and almost no enteral nutrition during TH. NICU1 with the greater amount of enteral nutrition on day 1 had an earlier start of enteral nutrition and an earlier timepoint until newborns received full enteral feeds. The feeding regime was independent of the level of encephalopathy. Similar outcomes were reported in a study from Florida, which showed that minimal enteral nutrition during $\mathrm{TH}$ led to a reduced time until newborns were fully orally fed and a reduced length of hospital stay [6]. Other benefits of enteral nutrition during $\mathrm{TH}$, such as higher survival to discharge, shorter time of parenteral nutrition, and earlier breastfeeding in comparison to children without enteral feeding during TH, were reported [19]. In contrast, a study from U.K. and Sweden reported that delayed enteral feeding did not alter the time to full enteral feeding, and children with delayed enteral feeds had a shorter median length of hospital stay than children with an early onset of enteral nutrition [5].

Although different amounts of enteral and total fluids were given in our study between NICU 1 and 2, we observed no rise in complication rate in either of the NICUs. In addition, a retrospective study from 2021 compared outcomes in infants who were fed versus not fed enterally during therapeutic hypothermia. They showed that the incidence of NEC and late-onset infection was reduced in children receiving feeding during TH [19].

It is also important to note that different factors influence the administration of enteral feedings. The administration was significantly different in severe and mild Sarnat scores, as well as in children with and without mechanical ventilation. The appearance of seizures and the lowest $\mathrm{pH}$ value after birth did not significantly influence the amount of enteral fluid supply. However, this might be due to the relatively low sample size in this study. Opioid therapy during TH might also have an impact on the restrictive approach to enteral feeding. Opioids such as morphine were given generously during TH to reduce the newborns' stress levels and improve the effectiveness of TH [20]. Morphine decreases intestinal motility, inhibits gastric emptying, increases sphincter tone, and causes blockage of peristalsis [21]. It might increase the risk for complications such as NEC or transmigration peritonitis.

In our cohort, it is noticeable that the high dose of morphine during $\mathrm{TH}$ was rapidly reduced after $\mathrm{TH}$ was finished (day 4 ) because the children were no longer exposed to the stress of TH. At the same time, enteral feeding was strongly increased $(30 \%)$ in all NICUs. In our cohort, NICU 1 used a much lower morphine dose and a much higher dose of enteral as well as total nutritional fluid intake compared to NICU 2, which shows that opioid therapy could be a possible reason for a more restrictive enteral feeding.

Although we revealed large differences in the amount of feeding in the different NICUs, it remains clear that all centers fed the neonates during TH without developing NEC or other morbidities, such as sepsis. 
The study has several limitations. A major limitation is the relatively small sample size and that we could not differentiate parenteral fluid supply based on its nutritional composition. A second limitation is the retrospective design. There were no existing feeding protocols. Therefore, it was not possible for us to retrace individual decisions of the clinicians regarding the amount of nutritional supply, choice of medication, sedation, etc. A third limitation is that newborns with mild encephalopathies were included in the study. The decision, why newborns with Sarnat 1 scores were cooled, was based on individual clinical decision making. Another limitation was that other factors that we did not investigate, such as liver function, also could have a meaningful influence on the tolerability of enteral nutrition. A final limitation is the missing long-term outcome data, e.g., the Bayley Scales of Infant Development (BSID). Nevertheless, it is important to obtain insight into the similarities and differences of the feeding regimes of four large NICUs in Germany, especially because there are no clear guidelines and the data on feeding regimens is scarce.

\section{Conclusions}

Comparing TH management in four major level I NICUs in Germany, we observed variability in the feeding regime. We conclude that early nutrition and especially early enteral nutrition is possible without increasing the rate of complications during TH. Further prospective studies are needed analyzing the influence of perinatal asphyxia and $\mathrm{TH}$ on feeding practices and, therefore, possible gut microbiome composition in cooled asphyxiated newborns.

Author Contributions: Conceptualization, M.M. and H.S.; Data curation, M.M., M.R., S.G., A.S. and H.S.; Formal analysis, M.M.; Methodology, M.M. and H.S.; Project administration, M.M. and H.S.; Resources, M.M., M.R., S.G., A.S. and H.S.; Software, M.M. and H.S.; Supervision, H.S.; Validation, M.M., M.R., S.G., A.S. and H.S.; Writing—original draft, M.M.; Writing—review and editing, M.M., A.S., T.H., U.F.-M., A.H., T.P., P.J. and H.S. All authors have read and agreed to the published version of the manuscript.

Funding: This research received no external funding.

Institutional Review Board Statement: The study was conducted according to the guidelines of the Declaration of Helsinki and approved by the Institutional Ethics Committee of Heinrich Heine University Duesseldorf (protocol code 2018-270-ProspDEuA and 2018-270-1, date of approval 20 March 2019 and 5 August 2019, respectively), University Duisburg Essen (protocol code 18-8191-BO and 19-8556-BO, date of approval 7 June 2018 and 27 February 2019, respectively), and University Oldenburg (protocol code 2019-110, date of approval 22 October 2019, respectively).

Informed Consent Statement: Patient consent was waived due to the retrospective design of the study.

Data Availability Statement: Data can be accessed and is available from the authors.

Conflicts of Interest: The authors declare no conflict of interest.

\section{References}

1. Glass, H.C. Hypoxic-Ischemic Encephalopathy and Other Neonatal Encephalopathies. Continuum 2018, 24, 57-71. [CrossRef] [PubMed]

2. Jacobs, S.E.; Morley, C.J.; Inder, T.E.; Stewart, M.J.; Smith, K.R.; McNamara, P.J.; Wright, I.M.R.; Kirpalani, H.M.; Darlow, B.A.; Doyle, L.W.; et al. Whole-Body Hypothermia for Term and Near-Term Newborns with Hypoxic-Ischemic Encephalopathy: A Randomized Controlled Trial. Arch. Pediatr. Adolesc. Med. 2011, 165, 692-700. [CrossRef] [PubMed]

3. Zingg, W.; Tomaske, M.; Martin, M. Risk of parenteral nutrition in neonates-An overview. Nutrients 2012, 4, 1490-1503. [CrossRef] [PubMed]

4. Hazeldine, B.; Thyagarajan, B.; Grant, M.; Chakkarapani, E. Survey of nutritional practices during therapeutic hypothermia for hypoxic-ischaemic encephalopathy. BMJ Paediatr. Open 2017, 1, e000022. [CrossRef] [PubMed]

5. Thyagarajan, B.; Tillqvist, E.; Baral, V.; Hallberg, B.; Vollmer, B.; Blennow, M. Minimal enteral nutrition during neonatal hypothermia treatment for perinatal hypoxic-ischaemic encephalopathy is safe and feasible. Acta Paediatr. 2015, 104, 146-151. [CrossRef] [PubMed] 
6. Chang, L.L.; Wynn, J.L.; Pacella, M.J.; Rossignol, C.C.; Banadera, F.; Alviedo, N.; Vargas, A.; Bennett, J.; Huene, M.; Copenhaver, N.; et al. Enteral Feeding as an Adjunct to Hypothermia in Neonates with Hypoxic-Ischemic Encephalopathy. Neonatology 2018, 113, 347-352. [CrossRef] [PubMed]

7. Morgan, J.; Young, L.; McGuire, W. Delayed introduction of progressive enteral feeds to prevent necrotising enterocolitis in very low birth weight infants. Cochrane Database Syst. Rev. 2014, 2014, CD001970. [CrossRef] [PubMed]

8. Rowlands, B.J.; Gardiner, K.R. Nutritional modulation of gut inflammation. Proc. Nutr. Soc. 1998, 57, 395-401. [CrossRef] [PubMed]

9. Konnikova, Y.; Zaman, M.M.; Makda, M.; D'Onofrio, D.; Freedman, S.D.; Martin, C.R. Late Enteral Feedings Are Associated with Intestinal Inflammation and Adverse Neonatal Outcomes. PLoS ONE 2015, 10, e0132924. [CrossRef] [PubMed]

10. Zanelli, S.; Buck, M.; Fairchild, K. Physiologic and pharmacologic considerations for hypothermia therapy in neonates. J. Perinatol. 2011, 31, 377-386. [CrossRef] [PubMed]

11. Giannakis, S.; Ruhfus, M.; Markus, M.; Stein, A.; Hoehn, T.; Felderhoff-Mueser, U.; Sabir, H. Mechanical Ventilation, Partial Pressure of Carbon Dioxide, Increased Fraction of Inspired Oxygen and the Increased Risk for Adverse Short-Term Outcomes in Cooled Asphyxiated Newborns. Children 2021, 8, 430. [CrossRef] [PubMed]

12. Ruhfus, M.; Giannakis, S.; Markus, M.; Stein, A.; Hoehn, T.; Felderhoff-Mueser, U.; Sabir, H. Association of Routinely Measured Proinflammatory Biomarkers With Abnormal MRI Findings in Asphyxiated Neonates Undergoing Therapeutic Hypothermia. Front. Pediatr. 2021, 9, 624652. [CrossRef] [PubMed]

13. Toet, M.C.; Hellström-Westas, L.; Groenendaal, F.; Eken, P.; de Vries, L.S. Amplitude integrated EEG 3 and 6 hours after birth in full term neonates with hypoxic-ischaemic encephalopathy. Arch. Dis. Child. Fetal Neonatal Ed. 1999, 81, F19-F23. [CrossRef] [PubMed]

14. Jacobs, S.E.; Berg, M.; Hunt, R.; Tarnow-Mordi, W.O.; Inder, T.E.; Davis, P.G. Cooling for newborns with hypoxic ischaemic encephalopathy. Cochrane Database Syst. Rev. 2013, 2013, CD003311. [CrossRef] [PubMed]

15. Azzopardi, D.V.; Strohm, B.; Edwards, A.D.; Dyet, L.; Halliday, H.L.; Juszczak, E.; Kapellou, O.; Levene, M.; Marlow, N.; Porter, E.; et al. Moderate hypothermia to treat perinatal asphyxial encephalopathy. N. Engl. J. Med. 2009, 361, 1349-1358. [CrossRef] [PubMed]

16. Flemmer, A.W.; Maier, R.F.; Hummler, H. Behandlung der neonatalen Asphyxie unter besonderer Berücksichtigung der therapeutischen Hypothermie. Klin. Pädiatrie 2014, 226, 29-37. [CrossRef] [PubMed]

17. Austin, T.; Ganado, C.C.; Clarke, P.; O'Hare, S.; Merchant, N.; Vakharia, B.; Waltson, F. Guidelines for Management of Infants with Suspected Hypoxic Ischaemic Encephalopathy (HIE); Clinical Guideline; NHS EOE Neonatal ODN: Cambridge, UK, 2016.

18. Neu, J.; Walker, W.A. Necrotizing enterocolitis. N. Engl. J. Med. 2011, 364, 255-264. [CrossRef] [PubMed]

19. Gale, C.; Longford, N.T.; Jeyakumaran, D.; Ougham, K.; Battersby, C.; Ojha, S.; Dorling, J. Feeding during neonatal therapeutic hypothermia, assessed using routinely collected National Neonatal Research Database data: A retrospective, UK population-based cohort study. Lancet Child Adolesc. Health 2021, 5, 408-416. [CrossRef]

20. Gundersen, J.K.; Chakkarapani, E.; Jary, S.; Menassa, D.A.; Scull-Brown, E.; Frymoyer, A.; Walløe, L.; Thoresen, M. Morphine and fentanyl exposure during therapeutic hypothermia does not impair neurodevelopment. EClinicalMedicine 2021, $36,100892$. [CrossRef] [PubMed]

21. Khansari, M.; Sohrabi, M.; Zamani, F. The Useage of Opioids and their Adverse Effects in Gastrointestinal Practice: A Review. Middle East J. Dig. Dis. 2013, 5, 5-16. [PubMed] 